\title{
Development And Characterisation Of Integrated Microfluidics On Waveguide-Based Photonic Platforms Fabricated From Hybrid Materials
}

\author{
Robert Copperwhite \\ National Centre for Sensor Research, School of Physical Sciences, Dublin City University \\ Mary O'Sullivan \\ National Centre for Sensor Research, School of Physical Sciences, Dublin City University \\ Catherine Boothman \\ National Centre for Sensor Research, School of Physical Sciences, Dublin City University
}

See next page for additional authors

Follow this and additional works at: https://arrow.tudublin.ie/cenresart

Part of the Physical Sciences and Mathematics Commons

\section{Recommended Citation \\ Oubaha, M. et al. (2011) Development And Characterisation Of Integrated Microfluidics On Waveguide- Based Photonic Platforms Fabricated From Hybrid Materials, Microfluid Nanofluid (2011) 11:283-296 doi:10.1007/s10404-011-0795-4}

This Article is brought to you for free and open access by the Crest: Centre for Research in Engineering Surface Technology at ARROW@TU Dublin. It has been accepted for inclusion in Articles by an authorized administrator of ARROW@TU Dublin. For more information, please contact arrow.admin@tudublin.ie, aisling.coyne@tudublin.ie,gerard.connolly@tudublin.ie. Funder: Enterprise Ireland

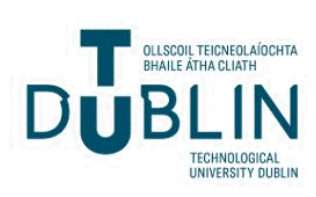




\section{Authors}

Robert Copperwhite, Mary O'Sullivan, Catherine Boothman, Arnaud Gorin, Colette McDonagh, and mohamed oubaha

This article is available at ARROW@TU Dublin: https://arrow.tudublin.ie/cenresart/54 


\title{
Development and characterisation of integrated microfluidics on waveguide-based photonic platforms fabricated from hybrid materials
}

\author{
Robert Copperwhite $\cdot$ Mary O'Sullivan • \\ Catherine Boothman • Arnaud Gorin · \\ Colette McDonagh $\cdot$ Mohamed Oubaha
}

Received: 21 December 2010/Accepted: 21 March 2011/Published online: 19 April 2011

(C) Springer-Verlag 2011

\begin{abstract}
This article reports on a detailed investigation of sol-gel processed hybrid organic-inorganic materials for use in lab-on-a-chip (LoC) applications. A particular focus on this research was the implementation of integrated microfluidic circuitry in waveguide-based photonic sensing platforms. This objective is not possible using other fabrication technologies that are typically used for microfluidic platforms. Significant results on the surface characterisation of hybrid sol-gel processed materials have been obtained which highlight the ability to tune the hydrophilicity of the materials by careful adjustment of material constituents and processing conditions. A proof-of-principle microfluidic platform was designed and a fabrication process was established which addressed requirements for refractive index tuning (essential for waveguiding), bonding of a transparent cover layer to the device, optimized sol-gel deposition process, and a photolithography process to form the microchannels. Characterisation of fluid flow in the resulting microchannels revealed volumetric flow rates between 0.012 and $0.018 \mu \mathrm{l} / \mathrm{min}$ which is characteristic of capillary-driven fluid flow. As proof of the integration of optical and microfluidic functionality, a microchannel was fabricated crossing an optical waveguide which demonstrated that the presence of optical waveguides does not significantly disrupt capillary-driven fluid flow. These results represent the first comprehensive evaluation of photocurable hybrid sol-gel
\end{abstract}

R. Copperwhite $(\bowtie) \cdot$ M. O'Sullivan · C. Boothman ·

A. Gorin · C. McDonagh · M. Oubaha

National Centre for Sensor Research, School of Physical

Sciences, Dublin City University, Dublin 9, Ireland

e-mail: robert.copperwhite@dcu.ie

C. McDonagh

Biomedical Diagnostics Institute, Dublin City University, Dublin 9, Ireland materials for use in waveguide-based photonic platforms for lab-on-a-chip applications.

Keywords Integrated microfluidics - Microfluidics .

Optical sensor - Biosensor - Photocurable hybrid sol-gel · Oxygen plasma

\section{Introduction}

As the proliferation of lab-on-a-chip (LoC) devices increases in the coming year, a key challenge remains in (i) the integration of optical waveguide-based detection platforms with microfluidic sample handling, and (ii) the large-scale fabrication of such devices at low cost. These requirements cannot be satisfied by the usual methods for microfluidic platform fabrication (e.g., hot embossing, micromilling) due to the heterogeneous refractive index contrast required by optical waveguides. To address this, a new fabrication process based on photocurable hybrid solgel materials is proposed which leverages the possibility to (i) easily tune refractive index, (ii) tune the surface characteristics of the material via the highly tunable sol-gel process, and (iii) directly photopattern the material by a low-cost photolithography process.

Photocurable hybrid sol-gel materials have a strong track record for the fabrication of planar optical waveguide-based platforms for telecommunications (Kribich et al. 2004; Porque et al. 2000) and more recently sensing (Kribich et al. 2005). Indeed, commercialised by Kloe (Montpelier, France) this technology has enjoyed significant growth and commercial success over the past decade. Similar to optical fibres, planar waveguides are generally composed of a core region in which light propagates, surrounded by a protective layer of lower refractive index. 
The key innovation reported here is the fabrication and characterization of integrated microfluidic circuitry (for fluid delivery) by the direct photopatterning of the lower refractive sol-gel protective layer which encapsulates the waveguide(s). Using this concept, a prototype biosensor platform integrating optical detection and microfluidic sample processing is envisaged, in which planar waveguides could be used for evanescent wave excitation of fluorescence from fluorescently labelled target biomolecules that are specifically captured on the waveguide surface via a biorecognition element (e.g., antibodies or aptamers). The use of photocurable hybrid sol-gel materials in an industrially compatible photolithography process (based on selective UV exposure through a photomask) is an attractive means of achieving this objective.

The sol-gel process is a low temperature, low pressure method to synthesise thin film materials which can be deposited by standard thin film deposition techniques such as spin- (Birnie 2004), dip- or spray-coating (Hwang et al. 2010). This synthesis route affords a great deal of control over the material composition via the correct choice of chemical precursors and synthesis conditions, resulting in a class of materials that are highly tunable. Multiple functionalities are often incorporated in a single hybrid material, which can serve the needs of diverse applicationsranging from electrochromic coatings for windows (Avellaneda et al. 2002; Avendano et al. 2006; Karuppasamy and Subrahmanyam 2007), corrosion protection (Varma et al. 2010; Hamdy 2006), porous sensor membranes for gas phase sensing (Higgins et al. 2008; Lei et al. 2006), entrapment of biomolecular probes for biosensors (Besanger and Brennan 2006; Gupta and Chaudhury 2007). Compared to pure organic polymers, sol-gel processed hybrid materials have advantages linked not only to their superior tunability as outlined above, but also linked to the production of interpenetrating, covalently bonded organic and inorganic networks within a single material, permitting the synthesis of materials with unique combinations of properties. Furthermore, the presence of a strong and rigid inorganic network formed through the hydrolysis and condensation reactions of the hybrid precursors confers superior long-term thermal stability compared to purely organic polymer materials (Zhang et al. 2004; Jiang and Kakkar 1999; Buso et al. 2009). This high degree of tunability offers the potential to satisfy a diverse range of material requirements.

With respect to fabrication processes, microfluidic platforms are typically fabricated by etching channels in glass(Hannes et al. 2008; Iliescu 2006), injection molding (Edwards et al. 2002), hot embossing (Becker and Heim 2000) and micromilling (Mescher et al. 2009) of polymers or soft lithography processes (Fujii 2002). However, with regard to the integration of microfluidics with optical platforms based on optical waveguides, these fabrication processes struggle to satisfy the requirements demanded by optical waveguides. The main requirement for waveguide fabrication is refractive index tunability so that a highrefractive index waveguide core is surrounded by a lower refractive index cladding material. It is also highly desirable that the process is industrially compatible and amenable to high volume low cost manufacturing. It has been demonstrated in many papers that these requirements are easily achieved using photocurable hybrid sol-gel materials in a photolithography process (Najafi et al. 1998; Coudray et al. 1998; Copperwhite et al. 2008; Oubaha et al. 2006; Oubaha et al. 2005).

The main focus of this article is to evaluate the feasibility of integrating microfluidics sample delivery on waveguide-based optical platforms based on photopatternable hybrid sol-gel materials. In order to achieve this the authors have carried out a systematic investigation which includes; (i) synthesis and evaluation of a range of material compositions focusing particularly on the wettability of thin film surfaces before and after oxygen plasma surface modification, (ii) identification of optimum material for microfluidic channel design and fabrication and subsequent evaluation of capillary-driven fluid flow, (iii) optical characterization and fabrication of a proof of principle optofluidic structure. To the knowledge, this is the first time that optical waveguide-based platforms with integrated microfluidic sample handling have been fabricated using photocurable hybrid sol-gel processed materials.

\section{Materials and methods}

\subsection{Sol-gel material compositions}

A range of hybrid organic-inorganic sol-gel material formulations were investigated for use in microfluidic channel fabrication. The materials were based on a photocurable sol-gel material which has been employed by the authors in previous publications for optical waveguide fabrication (Copperwhite et al. 2008). The hybrid sol-gel materials were based on the formation of a stable and homogeneous sol obtained from the mixture of a photosensitive organically modified silicate, the hybrid precursor 3-methacryloxypropyltrimethoxysilane (MAPTMS, Assay 99\% in methanol, Aldrich), zirconium (IV) $n$-propoxide $\left(\mathrm{Zr}(\mathrm{OPr})_{4}\right.$, Assay $\sim 70 \%$ in propanol, Aldrich) and methacrylic acid (MAA, $\mathrm{C}_{4} \mathrm{H}_{6} \mathrm{O}_{2}$, Assay $>98 \%$, Aldrich), a chelating agent, which is necessary to stabilize the zirconium $(\mathrm{Zr})$ precursor in the presence of strong nucleophilic groups such as water. Owing to the difference in reactivity of each of the alkoxide precursors present in the sols, the synthesis 
demands a three-step process: (1) pre-hydrolysis of the MAPTMS and complexation of ZPO with a chelating agent (2) addition of the pre-hydrolyzed organoalkoxysilane to the zirconate complex and (3) hydrolysis of the mixture. MAPTMS is hydrolyzed employing an aqueous $\mathrm{HCl} 0.1 \mathrm{~N}$ solution in a 1.00:0.75 ratio. As MAPTMS and water are not miscible, the hydrolysis is initially performed in a heterogeneous way. The progressive production of methanol issued from the hydrolysis of MATPMS allows the miscibility of all species present in solution after $20 \mathrm{~min}$ of reaction. In parallel, the ZPO precursor was chelated by MAA, in order to reduce its reactivity with water, thus avoiding precipitation. After $45 \mathrm{~min}$ of reaction, the partially hydrolyzed MAPTMS was slowly added to the zirconate complex. Following $5 \mathrm{~min}$ of reaction, water $(\mathrm{pH}$ 7) was added dropwise to the mixture to complete the hydrolysis of a predefined percentage of the total alkoxide groups. A more detailed account of the sol-gel synthesis can be found in a previous paper by the authors (Oubaha et al. 2003). A photoinitiator was added to the final sol prior to use in order to make the materials photopolymerisable. For all materials in this article, the photoinitiator Irgacure 184 (Ciba Speciality Chemicals) was chosen for these experiments due to its high stabilility in zirconiumbased sol-gel materials (Versace et al. 2008), and the concentration was fixed at $5 \mathrm{~mol} \%$ (with respect to the methacrylate groups of MAPTMS). Photoinitiator was left to stir for $1 \mathrm{~h}$ and then the sol was filtered through a $0.2 \mu \mathrm{m}$ filter prior to use.

The first study conducted in this study involved the synthesis of the "Group A" materials (Table 1), in which the molar ratio of the three main components of the material were varied in order to examine the influence of each component on the surface properties of the final material. In the context of capillary-driven fluid flow, the main surface property of interest were the wettability of the surface (as measured by the water contact angle (CA)), and the evolution of wettability over time. To this end the authors have changed the relative proportions of ZPO and MAA whilst keeping the amount of MAPTMS constant. In addition, for each of these formulations 2 hydrolysis rates were employed in the sol-gel synthesis to assess the influence that the condensation degree and the eventual increase of the pendant hydroxyl groups provokes on the surface properties.

Having extracted some conclusions from the Group A materials, another group of materials (Group B) was synthesized to investigate in greater depth the variation of surface properties derived from varying the relative proportions of the zirconate complex against the organosilane. For these experiments hydrolysis was fixed at $50 \%$, and the ZPO:MAA ratio was maintained at $1: 1$, as shown in Table 2.
Table 1 Summary of the Group A material formulations

\begin{tabular}{ll}
\hline Group A & \\
\hline (MAPTMS:Zr(OPr) $4: M A A)$ & \\
\hline $50 \%$ Hydrolysis & $100 \%$ Hydrolysis \\
\hline $10: 2: 2$ & $10: 2: 2$ \\
$10: 2: 4$ & $10: 2: 4$ \\
$10: 4: 4$ & $10: 4: 4$ \\
$10: 4: 8$ & $10: 4: 8$ \\
\hline
\end{tabular}

Table 2 Summary of the Group B material formulations

Group B

(MAPTMS:Zr(OPr) $\left.{ }_{4}: \mathrm{MAA}\right)$

10:1:1

$10: 2: 2$

$10: 3: 3$

$10: 4: 4$

\subsection{Material characterisation}

\subsubsection{Sample preparation and surface modification/ storage}

Characterization of materials was first done on planar, unstructured thin films in order to assess the optical properties (refractive index) and surface properties (wettability) of materials with and without surface modification. Thin film samples were prepared on silicon substrates by spincoating (Laurell WS-650S) the sol-gel material at a speed of $1000 \mathrm{rpm}$ under an alcohol saturated environment. The purpose of spin-coating in an alcohol saturated environment was to control the kinetic of evaporation of the solvents during the deposition process, thus obtaining homogeneous and high optical quality thin films.

Surface modification was done via oxygen plasma treatment in order to increase the wettability of the surfaces. It is well-known that hydrophilic channel surfaces are desirable for applications in microfluidics as they support pump-free fluid flow under capillary action (Zhu and Petkovic-Duran 2010). Surfaces were modified by exposure to oxygen plasma for 5 min (Harrick Plasma PDC-002, $30 \mathrm{~W}, 0.1$ Torr $\mathrm{O}_{2}, 3 \mathrm{sccm}$ ).

\subsubsection{Contact angle measurements}

Static water contact angles (CA) on hybrid sol-gel surfaces (unmodified and plasma treated) were measured by the sessile drop method using deionized water, under air at room temperature using a video-based optical CA measuring device (FTA200, First Ten Ångstroms) with an electronic syringe unit. Uniform drops of $2 \mu \mathrm{l}$ were 
dispensed onto the surface, and following a $10 \mathrm{~s}$ delay to allow the drops to stabilize, digital images of the drops were taken. Drop images were analysed using the FTA32 Video 2.0 software to yield the water contact angle.

\subsubsection{Refractometry}

Refractive index values for thin films formed from the Group B series of materials were determined using the prism coupling method at $632.8 \mathrm{~nm}$ (Metricon 2010 Prism Coupler). Using this technique, a measurement accuracy of \pm 0.001 is routinely achieved, which was taken to be the measurement uncertainty.

\subsubsection{Hydrophobic recovery}

Group B samples (unmodified and plasma treated) were stored in ambient conditions at room temperature with exposure to air over a 26 day period. The effect of these conditions on surface properties was evaluated by measuring the $\mathrm{CA}$ at regular intervals. In this way, hydrophobic recovery of samples could be tracked as a function of sample composition in order to determine the optimum material to support capillary-driven fluid flow in microfluidic channels. A more in depth study of the kinetics of hydrophobic recovery in the first $6 \mathrm{~h}$ after plasma treatment was conducted using the 10:2:2 material due to its combination of material characteristics that render it attractive for integrated microfluidic platform fabrication. In addition, another 10:2:2 sample was stored under nitrogen (in a flow cell under a constant flow of nitrogen) in order to assess whether storage media could influence or lengthen the period of time over which surface pre-treatment of the material was effective.

\subsection{Microfluidic channel designs}

Microfluidic channels were designed to test the suitability of the photocurable hybrid sol-gel material systems for employment in the fabrication of integrated microfluidic waveguide-based platforms. Designs were drafted using the mask design module (Thor interface) of the OlympIOs Integrated Optics Software (C2 V, Netherlands). Low-cost film masks with $20 \mu \mathrm{m}$ resolution were purchased from JD Photo-Tools, UK. A variety of microfluidic channels were designed including simple fixed width channels and more complex structures including serpentine channels. Wide channels containing micropillars arrays (of defined radius and separation) were also designed. Micropillars have two important roles in microfluidic channel operation: firstly, they prevent the PDMS cap layer from bonding to the bottom surface of the channel, which was a problem for wide channels which did not feature micropillars; secondly, they encourage fluid flow within a wide channel by means of capillary wicking as is observed in lateral flow platforms (Ho et al. 2008).

\subsection{Microfluidic channel fabrication process}

The photolithographic process developed for the fabrication of microfluidic channels is illustrated in Fig. 1. This process involved five main steps starting with a bare substrate material. A silicon wafer was used in this study due to the ability to conveniently divide up a full wafer by cleaving along crystallographic planes, but it is worth noting that other flat substrate materials such as glass or plastic are also suitable.

Step 1 The silicon wafer was first coated with a 'buffer layer' (BL) thin film which forms the bottom surface of the microfluidic channels. In this study a BL composed of the 10:2:2 material was chosen based on a combination of favourable surface and processing properties which will be examined in Sect. 3. The BL was spin-coated at $700 \mathrm{rpm}$ under an alcohol saturated environment and then fully stabilised (i.e., rendered insoluble in the alcoholic solvents used to develop the microfluidic channels in subsequent process steps) by exposure to UV light for $10 \mathrm{~min}$.

Step 2 In the final application (outlined in Sect. 1), the microfluidic channels are to be formed in the protective layer (PL) which encapsulates planar waveguides that are formed in a previous process step. For initial testing of microfluidic channels, the planar waveguides were not fabricated and the PL (same material composition as the BL) was directly deposited on top of the BL. The height of the microfluidic layer can be tuned by adjusting the spincoating speed employed in PL deposition. In this case, a spin-speed of $700 \mathrm{rpm}$ was used, yielding a PL of $8.9 \mu \mathrm{m}$ in height. The PL was pre-exposure baked (PEB) on a vacuum hot-plate for $60 \mathrm{~min}$ at $110^{\circ} \mathrm{C}$ to make the layer sufficiently dry for the subsequent photolithography step.

Step 3 Having allowed the wafer to cool to room temperature, microfluidic channels were patterned in the PL by exposure to UV light from a mask aligner (Karl Suss MA 1006) through a film mask placed on the PL surface. A UV exposure time of $240 \mathrm{~s}$ was employed to yield a high fidelity image.

Step 4 The pattern was developed by etching the UV exposed PL in 1-butanol, gentling agitating the wafer in a petri dish of the solvent for $20 \mathrm{~s}$, followed by drying in a $\mathrm{N}_{2}$ stream. To fully stabilize the PL after exposure and etching, the wafer was post-exposure baked for $1 \mathrm{~h}$ at $110^{\circ} \mathrm{C}$ in a convection oven.

Step 5 To seal the microfluidic channel a PDMS cap layer to was made by thoroughly mixing curing agent and PDMS prepolymer (SYLGARD 184 Silicone Elastomer Kit, Dow Corning, MI) in a 1:10 weight ratio. The 
Fig. 1 Microfluidic channel fabrication process

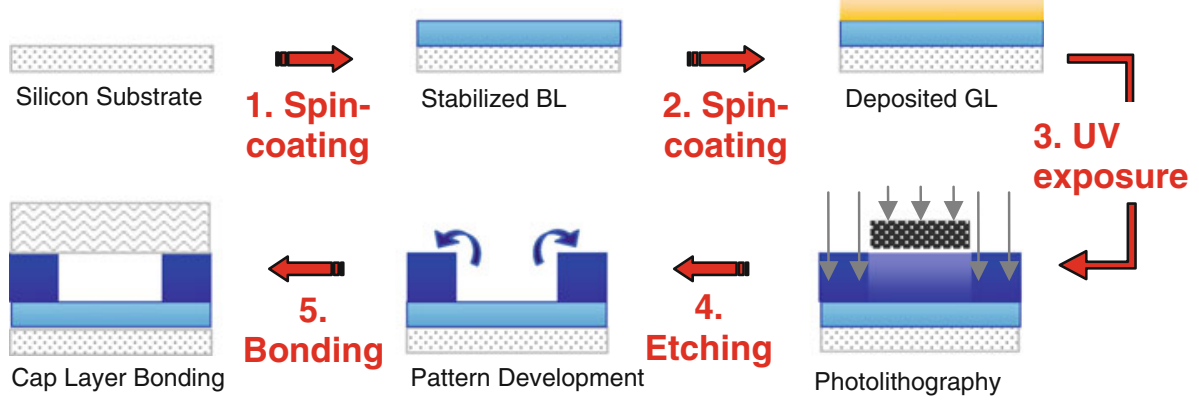

prepolymer mixture was degassed by sonication for $30 \mathrm{~min}$ to remove any air bubbles in the mixture and to ensure that a homogenous mixture was obtained. Then the liquid PDMS was cast against a silicon wafer to form a perfectly flat surface for bonding to the microfluidic channels. After $1 \mathrm{~h}$ drying in an over at $70^{\circ} \mathrm{C}$, the PDMS layer was peeled from the wafer surface and cut into rectangles with a blade. Access holes which acted as reservoirs for fluid were punched (Harris Uni-Core, Tip Diameter $3.5 \mathrm{~mm}$ ) into the PDMS. In order to assist bonding between the PL surface and the PDMS cap, both the microfluidic platform and the PDMS layer were oxygen plasma modified for $2 \mathrm{~min}$ as outlined in Sect. 2.2.1. This was done to aid bonding between the two materials, as it was previously shown that such treatment can aid in the formation of irreversible bonds between PDMS and glass substrates (Bhattacharya et al. 2005a, b).

Although, the study reported here is focused mainly on the assessment of the hybrid sol-gel material system for microfluidic channel fabrication as illustrated in Fig. 1, a proof of concept experiment was carried out in which photonic components such as optical waveguides were integrated with the fabrication of microfluidics channels as illustrated in Fig. 2. In step 3 of this process, the sol-gel guiding layer (GL) of slightly higher refractive index (composed of the 10:2.6:2.6 material as determined by refractive index analysis presented in Sect. 3.3) was spincoated at $800 \mathrm{rpm}$ as outlined previously to arrive at a layer of $6 \mu \mathrm{m}$ thickness. Channel optical waveguides were fabricated by UV exposure of this layer though a chrome photomask (step 4) using the mask aligner. Development of the patterned GL (step 5) was carried out by immersion in 1-butanol for $20 \mathrm{~s}$.

\subsection{Microfluidic channel characterisation}

\subsubsection{Optical microscopy, profilometry and SEM}

Images of fabricated microfluidic channels were captured in top view by an optical microscope (Olympus EX51 M) coupled to a CCD camera. Image capture software permits the dimensions of the structures to be measured with a resolution of $\pm 0.2 \mu \mathrm{m}$. Optical profilometry (Veeco Wyko NT1100 Optical Profilometer) was employed to measure the depth of channels prior to bonding with the PDMS cap layer. It was also used to characterize the surface roughness of the spin-coated hybrid sol-gel thin films. SEM measurements were carried out using a Hitachi S3400n Scanning Electron Microscope.
Fig. 2 Process for integration of microfluidic channels on integrated optical platforms

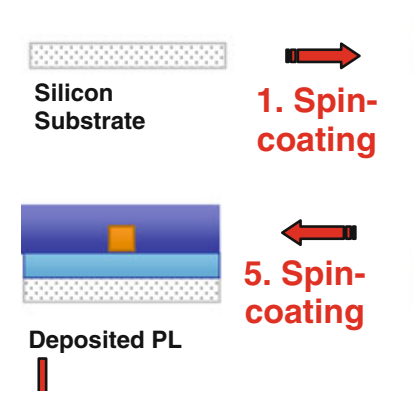

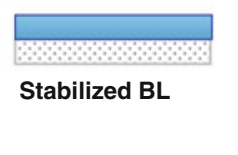

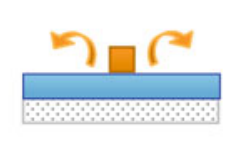

Pattern Development

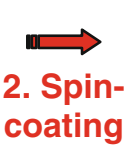

2. Spin-
coating

4.

Etching

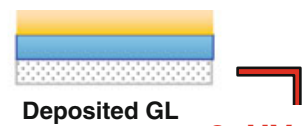

Deposited GL

3. UV exposure

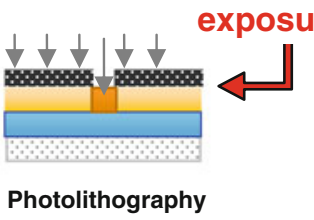

6. UV

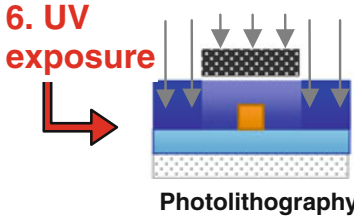

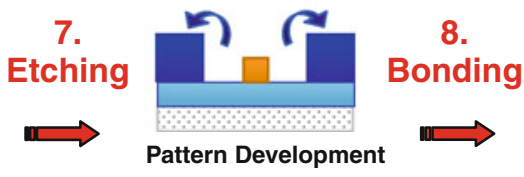

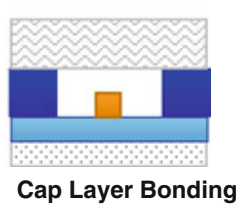




\subsubsection{Characterisation of capillary-driven flow}

In order to produce a fluid that was easily identifiable as it flowed through microfluidic channels, deionised water was mixed with $10 \% \mathrm{v} / \mathrm{v}$ of green or red food colouring (Goodall's, Ireland). Fluid was dropped into the reservoir created in the PDMS cap layer using a syringe and fluid flow could be observed immediately by viewing from the top through the transparent PDMS cap layer. Fluid flow in the channels was recorded by using a PC-interfaced video camera (JVC Color Video Camera, TK-C1480E) coupled to an optical microscope (Olympus EX51 M) fitted with a $4 \times$ objective lens. Using video editing software (AVS Video Editor 4.2), fluid flow rates were extracted from the acquired video files by monitoring the location of the fluid meniscus as a function of time. The volume of the channel filled at a particular time was quantified by multiplying the distance liquid had travelled in the channel at that time by the average cross-sectional area of the channels (evaluated by division of the calculated free volume by the length). Volumetric flow rates were determined from the slope of the data calculated by multiplying the flow rate of the interface by the channel cross-sectional.

\section{Results and discussion}

This section deals with the presentation of results from surface characterisation experiments, comparing the wettability of thin film surfaces composed of different materials, both with and without surface modification. Results concerning the effect of storage medium on surface wettability are also presented. The fabrication process developed for microfluidic channel fabrication is discussed with images of fabricated channels presented. Data related to capillary-driven fluid flow are presented and discussed. Finally, a proof of concept optofluidic structure was fabricated in which planar optical waveguides were integrated with microfluidic channels.

\subsection{Surface characterisation-effect of oxygen plasma modification}

Contact angle measurement data of 'Group A' materials are shown in Fig. 3. These measurements were carried out on both unmodified and oxygen plasma modified surfaces at regular intervals over a 6 day period. In Fig. 3a and c, measured CA values between 63 and $67^{\circ}$ can be observed for the unmodified surfaces composed of 100 and $50 \%$ hydrolysed materials, respectively. Within the error bars of the measurements, it is not possible to observe a significant CA difference between these materials, based either on hydrolysis or upon the length of time stored (varying between 0 and 6 days in ambient conditions).

However, inspection of the CA data displayed in Fig. 3b and $\mathrm{d}$ reveals that oxygen plasma modification causes considerable changes in the surface properties. Starting with data gathered directly after modification (0 days), it is clear that oxygen plasma modification results in a dramatic decrease of the CAs. Both 10:2:2 and 10:2:4 materials were rendered superhydrophilic with $\mathrm{CA}=2.9^{\circ}$, corresponding to an average CA decrease of $95 \%$ compared to the unmodified materials. The fact that this occurred for both 100 and 50\% hydrolysed materials, indicates that the level of hydrolysis of these materials has no significant effect on the wettability of the surfaces directly after oxygen plasma modification (day 0). Inspection of CA data from the higher zirconium content materials (10:4:4 and 10:4:8) reveals that these materials are also rendering hydrophilic by the oxygen plasma modification but to a lesser extent than the 10:2:2 and 10:2:4 materials. Furthermore, it can be seen that for these materials, the extent of hydrolysis of the material $(100 \%$ or $50 \%)$ is important, with the $50 \%$ hydrolysed material exhibiting a lower average CA $\left(\mathrm{CA}=7.2^{\circ} \rightarrow 89 \%\right.$ decrease $)$ than the $100 \%$ hydrolysed material $\left(\mathrm{CA}=14.8^{\circ} \rightarrow 76 \%\right.$ decrease $)$. From these data it is evident that a lower hydrolysis degree and a lower concentration of ZPO both result in more hydrophilic surfaces after oxygen plasma modification.

Comparison of the materials in terms of zirconium content offers a plausible explanation for observed differences in wettability. It has previously been demonstrated that polycondensation reactions of the silanol groups into irreversible siloxane groups are catalyzed by the inclusion of ZPO (Oubaha et al. 2003). The result is a significant increase in siloxane bonds which are well-known to be hydrophobic (Jaynes and Boyd 1991). The underlying mechanism that causes increased hydrophobicity for $100 \%$ compared to $50 \%$ hydrolysed materials is similar to that explained above for increased ZPO content. That is, if a larger quantity of water is added to a sol during synthesis, a larger amount of reactive metal hydroxyl groups $(\mathrm{SiOH}$ and $\mathrm{ZrOH}$ ) are generated ultimately leading to a more condensed material, i.e., containing a greater proportion of hydrophobic $\mathrm{Si}-\mathrm{O}-\mathrm{Si}$ (siloxane) and $\mathrm{Zr}-\mathrm{O}-\mathrm{Zr}$ oligomers.

Oxygen plasma modification of the surface of these materials is thought to generate silanol $(\mathrm{SiOH})$ groups which are known to be hydrophilic (Fois et al. 2008; Efimenko et al. 2002). The mechanism by which this occurs is likely to be the same as that occurring in the oxygen plasma treatment of polydimethylsiloxane (PDMS) which, similar to MAPTMS consists of repeating units of $\mathrm{Si}-\mathrm{O}-\left(\mathrm{CH}_{3}\right)_{2}$. In its pure state, MAPTMS has end groups composed of $\mathrm{Si}-\mathrm{O}-\left(\mathrm{CH}_{3}\right)_{3}$. NMR experiments carried out in a previous paper by one of the authors showed that after 
Fig. 3 Contact angle measurements of different solgel material compositions which are: a $100 \%$ hydrolysed and non-plasma treated, b $100 \%$ hydrolysed and oxygen plasma treated, c 50\% hydrolysed and non-plasma treated, d $50 \%$ hydrolysed and oxygen plasma treated (all compositions measured over a 6 day period). Material composition refers to the molar ratio of sol-gel constituents,

MAPTMS:ZrOPr 4 :MAA
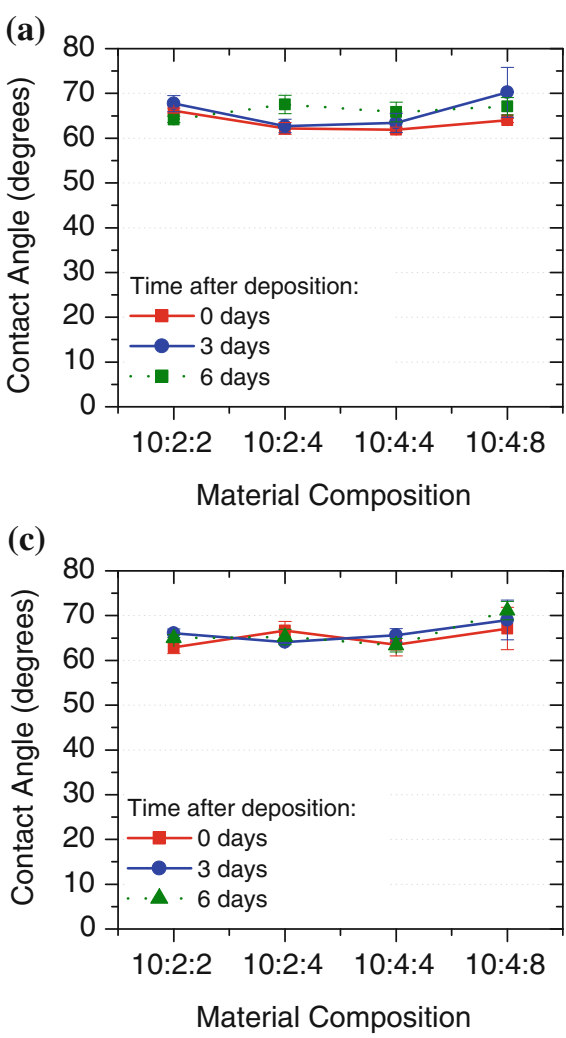

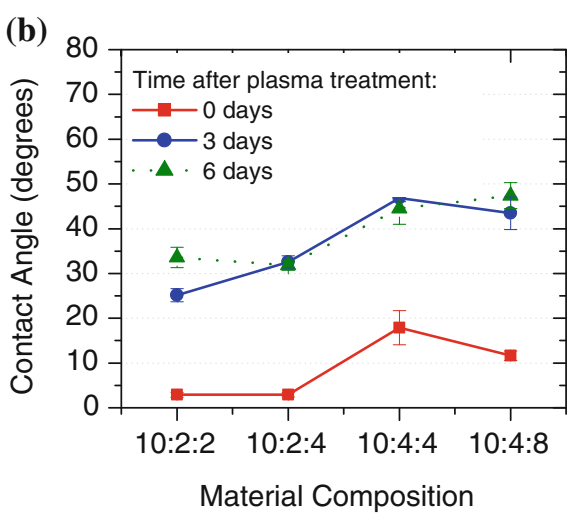

(d)

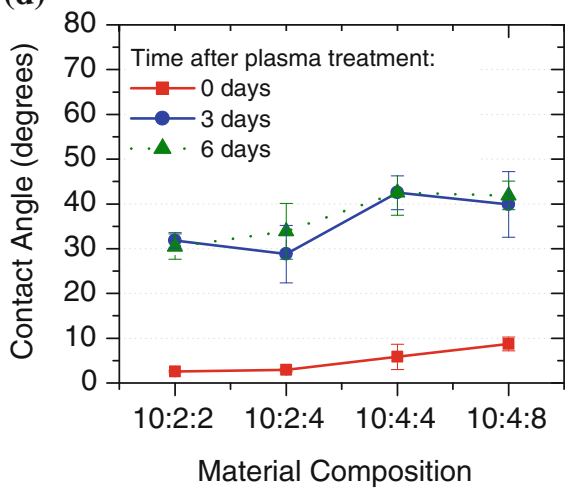

drying, the hybrid sol-gel material composed of a mixture of fully and partially condensed groups, including the presence of methoxy groups $\left(\mathrm{O}-\mathrm{CH}_{3}\right)$. Most research into PDMS indicates that exposure of the repeating units to oxygen plasma leads to the formation of silanol groups ( $\mathrm{Si}-$ $\mathrm{OH})$ which are developed at the expense of methyl groups (Bhattacharya et al. 2005a, b; Everaert et al. 1995).

From the above analyses, it is clear that to tune a hybrid sol-gel material system (such as the one proposed in this study) towards the formation of hydrophilic surfaces, one must carefully control the degree of hydrolysis of the material so that the tendency to form hydrophilic hydroxyl groups is balanced against the consumption of such groups in subsequent condensation reactions (which lead to hydrophobic surfaces). Furthermore, in materials where a transition metal (such as $\mathrm{Zr}$ ) is employed to enable refractive index control of an organosilane matrix, it is critical to account for the interaction between reactive groups in the sol, which can result in a dramatic increase of the material condensation, also leading to more hydrophobic properties.

\subsection{Hydrophobic recovery and kinetics}

In addition to CA data measured directly after thin film deposition/oxygen plasma modification, the data displayed in Fig. 3 also show the evolution of CA over a period of 2 and 6 days. For the unmodified samples analysed in Fig. 3a and $\mathrm{c}$, no significant change in CA can be observed after 2 and 6 days storage (ambient conditions). However, the oxygen plasma modified samples represented in Fig. $3 b$ and $d$, exhibited significant hydrophobic recovery after 2 and 6 days, whatever the hydrolysis rate employed. Owing to the scale of experimental uncertainty in the data it is difficult to compare the rates of hydrophobic recovery between materials. However, it is possible to quote an average increase in CA of $14.7^{\circ}$ (from day 0 to 2 ) and of $15.6^{\circ}$ (from day 0 to 6 ), which are not possible to distinguish within the experimental uncertainty. This suggests that the initial rate of hydrophobic recovery is fast, followed by another stage in the process which occurs at a much slower rate.

Also it is notable that within the error bars of the measurements taken, it is not possible to discern any significant change in behaviour depending on the amount of chelating agent linked to the zirconium atom. With regards to the effect of hydrolysis, it can be seen clearly from the 10:4:4/10:4:8 CA data that the average CA values for the $100 \%$ hydrolysed materials are $5^{\circ}$ higher than for the $50 \%$ hydrolysed materials, further supporting the explanation developed earlier concerning the effect of the hydrolysis rate employed on the wettability of the resulting surfaces.

In order to further highlight the relationship between the material composition and surface properties of this series 
of materials, another group of materials (Group B) containing a progressive increase of the ZPO content was synthesized. All group B materials were hydrolyzed to $50 \%$, and contained a ZPO:MAA ratio of $1: 1$. Data from the CA analysis of group $\mathrm{B}$ materials are presented in Fig. $4 \mathrm{a}$ for unmodified surfaces, and Fig. 4b for oxygen plasma modified surfaces.

It is clearly evident from these data that increasing ZPO content results in more hydrophobic surfaces after oxygen plasma treatment. Furthermore, it is clear that hydrophobic recovery of materials containing a lower ZPO concentration occurs at a slower rate, for example, after 26 days the 10:1:1 surface reached a CA of $40^{\circ}$ compared to $10: 4: 4$ which reached $66^{\circ}$.

The 10:2:2 material was subjected to a complete study of the kinetics of hydrophobic recovery as presented in Fig. 5. This material was chosen above the others synthesized due to its possession of favourable properties for photolithography including; ability to form a sufficiently dry surface which does not stick to a photomask, sufficient photopatternability; greater wettability than materials containing higher ZPO content. From the data, it is observable that hydrophobic recovery occurs in a two-stage process. In the first $6 \mathrm{~h}$ after oxygen plasma modification hydrophobic recovery is very rapid with an increase in CA of $2.7^{\circ} / \mathrm{h}$ (see inset in Fig. 5). The second stage in hydrophobic recovery is observed to take place $6 \mathrm{~h}$ after oxygen plasma modification, during which the rate of CA increase reduces to a lower level of $0.07^{\circ} / \mathrm{h}$ or $1.6^{\circ} /$ day. At this slower rate of change, the material returns to the value of an unmodified surface after 26 day of storage in ambient conditions. The rapid CA increase observed in stage one can be attributed to the rapid condensation of surface silanols to form siloxanes, which has been posited as one of the mechanisms of hydrophobic recovery in PDMS (Everaert et al. 1996). The second stage of hydrophobic recovery is attributed to the diffusion of lower molecular weight species from the bulk of the film onto the film surface, which is commonly thought to play the dominant

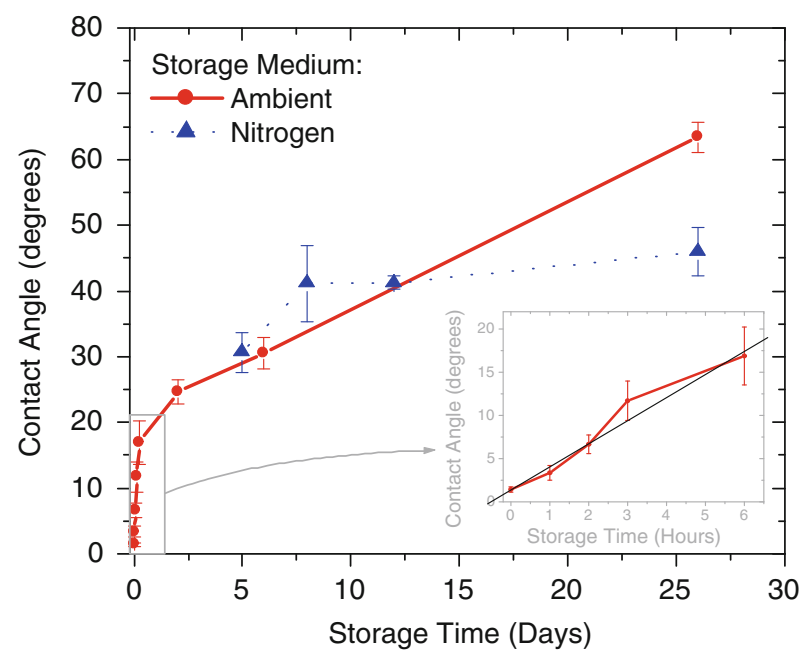

Fig. 5 Contact angle versus time of a 10:2:2 thin film showing hydrophobic recovery in ambient and nitrogen atmospheres. Inset shows zoomed in view of hydrophobic recovery from 0 to $6 \mathrm{~h}$ after oxygen plasma modification

role in hydrophobic recovery of a variety of pure polymer materials. This is valid for the hybrid sol-gel materials because it possesses a strong polymeric character due to the presence of organic groups throughout the material.

Also present in Fig. 5 are data which elucidate the role of storage medium on the hydrophobic recovery of surfaces. In this case, a similar 10:2:2 sample was stored in a nitrogen atmosphere for $\sim 3$ weeks. It can be seen from these data that the initial CA increase is similar to that of the sample stored in ambient conditions. However, the data point taken after 26 days shows that the CA of the surface has stabilised at $46^{\circ}$ which represents a CA increase of $4.5^{\circ}$ $\left(0.34^{\circ}\right.$ day), which cannot be distinguished from the value measured 14 days earlier within experimental uncertainty. Compared to the $\mathrm{CA}$ increase of the sample stored in ambient conditions over the same 14 day time period (CA increase of $27.3^{\circ} \rightarrow 1.9^{\circ}$ day), this result shows that storage of hybrid sol-gel surfaces in a nitrogen atmosphere can significantly slow down the rate of hydrophobic recovery.
Fig. 4 Contact angle of different materials at various times after deposition: a not oxygen plasma treated, b oxygen plasma treated. Material Composition refers to the molar ratio of the sol-gel constituents,

MAPTMS:ZrOPr 4 :MAAH
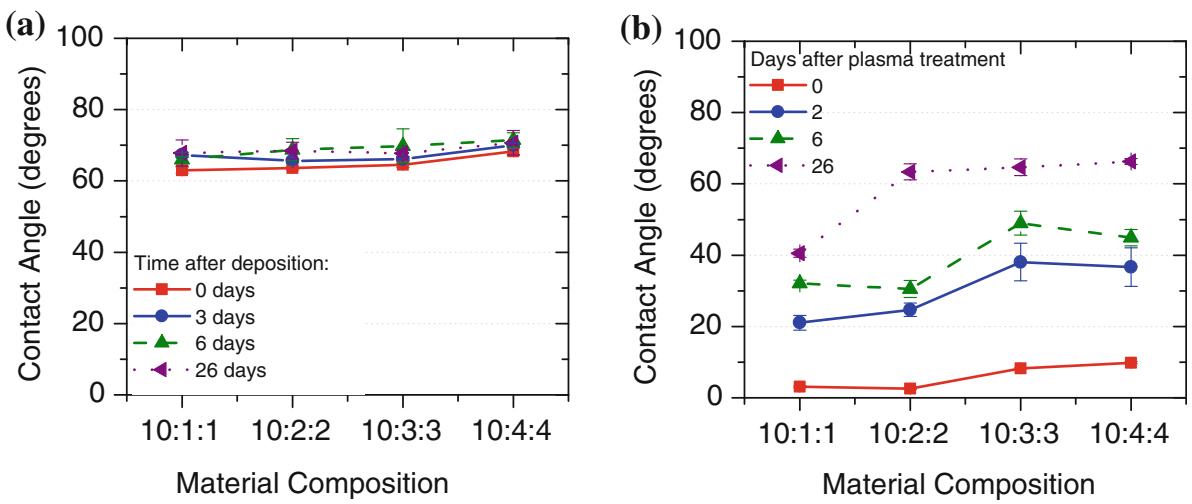
This is a promising result which has important implications for the industrial compatibility of these materials for fabrication of capillary driven platforms, and as such this observation will be explored further in a future paper. The CA analysis discussed in this section brings new understanding of the surface properties offered by photocurable sol-gel derived materials, with particular emphasis on tailoring the CA for application to microfluidic channel fabrication for lab-on-a-chip devices.

\subsection{Refractometry}

Refractometry of thin films formed from Group B (and some other intervening materials) is shown in Fig. 6, where thin film refractive index is displayed as a function of the MAPTMS:ZPO molar ratio (10:X). The refractive index of these materials is seen to increase linearly as the ZPO concentration is increased. Precise control over the refractive index of this material makes it suitable for employment for waveguide-based photonic platforms, as it permits the formation of a high-refractive index waveguide core (GL) surrounded by a lower refractive index protective layer (PL). In the case of the proof of concept structure outlined in Fig. 2, a refractive index difference of 0.004 between the GL and PL is required for single-mode waveguide operation at a wavelength of $635 \mathrm{~nm}$. Examination of Fig. 6 shows that for a PL with molar ratio of 10:2:2 between the main components, a GL composed of 10:2.6:2.6 achieves the required refractive index difference.

\subsection{Optimized sol-gel spin-coating process}

In order to exploit the developed sol-gel materials in an industrial process, a critical requirement is the ability to repeatedly coat highly uniform thin films. Therefore, a spincoating deposition process was developed which addressed

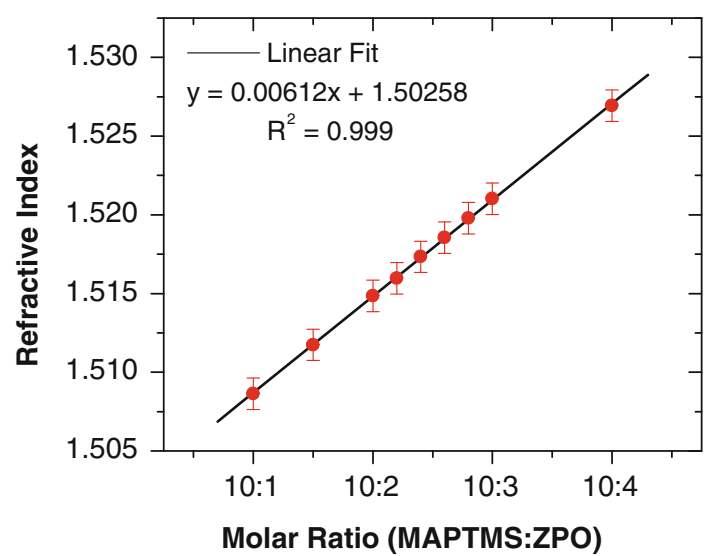

Fig. 6 Refractive index as a function of the molar ratio of MAPTMS:ZPO in a photocurable hybrid sol-gel material initial difficulties encountered with deposition in ambient conditions. In the initial stages, it was found that films coated in atmospheric conditions were often of poor quality with high levels of non-uniformity observed. In Fig. 7a, the surface profile of a film coated in atmospheric conditions is presented, from which an average roughness value of $\sim 717 \mathrm{~nm}$ was calculated. This is due to the uncontrolled evaporation of the solvents resulting in an immediate and heterogeneous drying of the thin film. To address this problem, the spin-coater was adapted to receive alcohol saturated $\mathrm{N}_{2}$ which was produced by flowing nitrogen through sealed flasks containing isopropanol (IPA). The vast improvement given by coating in the optimised setup is evident in the surface profile shown in Fig. 7b, from which an average surface roughness of $10.23 \mathrm{~nm}(98.6 \%$ decreases in roughness) was measured using the same measurement parameters. A high-pass filter was applied to these data to remove the contribution of waviness to the overall roughness value, yielding a roughness of 5.42 and $0.06 \mathrm{~nm}$ for the films coated in atmospheric and alcohol saturated environments, respectively ( $98.8 \%$ decreases in roughness). This shows that spin-coating in alcohol saturated conditions significantly reduces waviness of the coating without affecting the nanoscale surface roughness. The improvement in surface uniformity is attributed to the suppression of solvent evaporation from the coating by the IPA saturated $\mathrm{N}_{2}$. Also, the introduction of $\mathrm{N}_{2}$ into the spin-coater significantly reduces the levels of atmospheric humidity present during spincoating (relative humidity is typically reduced to $<20 \%$ ), thus inhibiting the formation of a surface gel layer during spinning by rapid hydrolysis and condensation reactions on the surface, which is another cause of coating defects (Birnie 2004).

\subsection{Characterisation of sol-gel microfabricated structures}

The microfluidic channels described by the mask design in Sect. 2.3 were fabricated according to the process described in detail in Sect. 2.4. The optical analysis of some fabricated structures is presented here along with images demonstrating fluid flow in microfluidic channels. A typical image of fluid flow in a straight channel is presented in Fig. $8 \mathrm{a}$ in which a parabolic liquid meniscus shape is observed, characteristic of capillary-driven flow in hydrophilic channels (Basabe-Desmonts et al. 2008). In addition to straight channels, serpentine channel patterns were also fabricated as shown in Fig. 8b, which demonstrates that more complex microfluidic structures can be manufactured using this material system in a simple photolithography process. Such serpentine structures are have been demonstrated to provide greatly improved mixing for Reynolds numbers from 6 to 70 (Liu et al. 2000). 

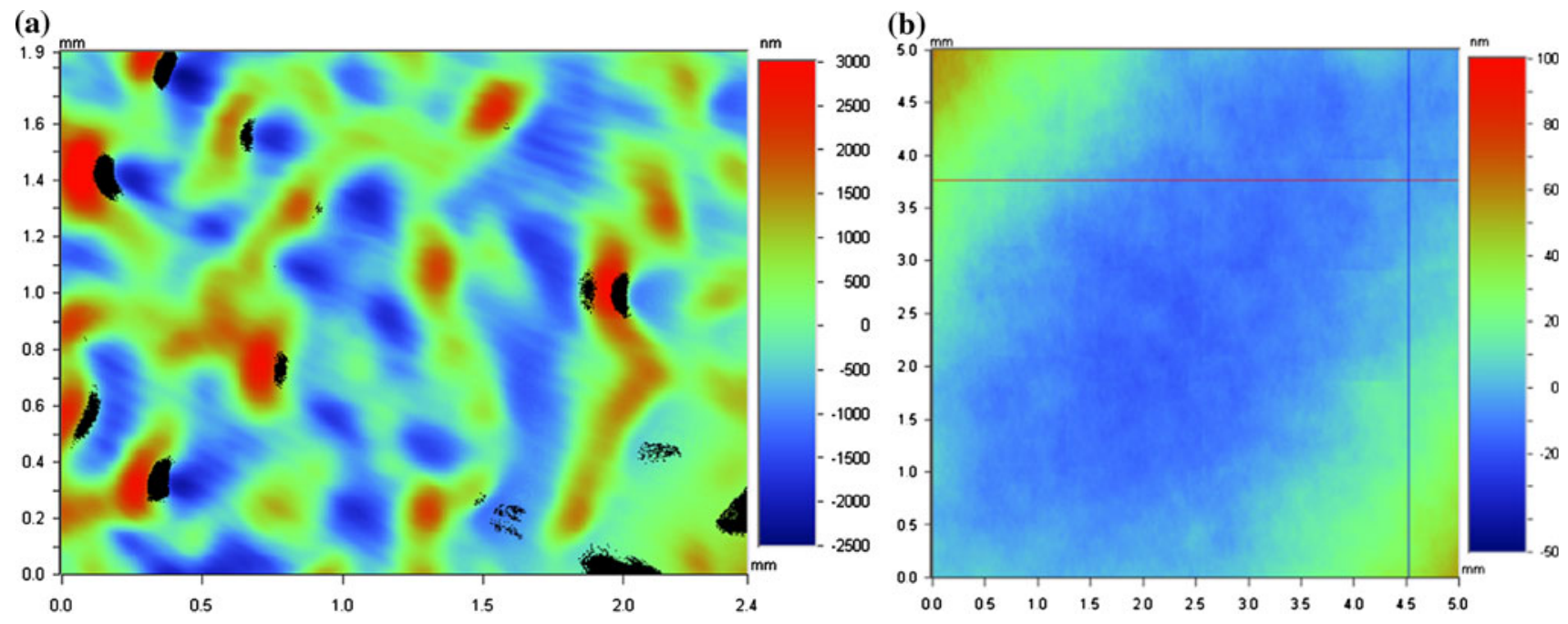

Fig. 7 Optical profilometry of hybrid sol-gel material spin coated in a ambient conditions, and $\mathbf{b}$ in an alcohol saturated environment

Fig. 8 Capillary driven fluid flow in: a straight, and b serpentine channels
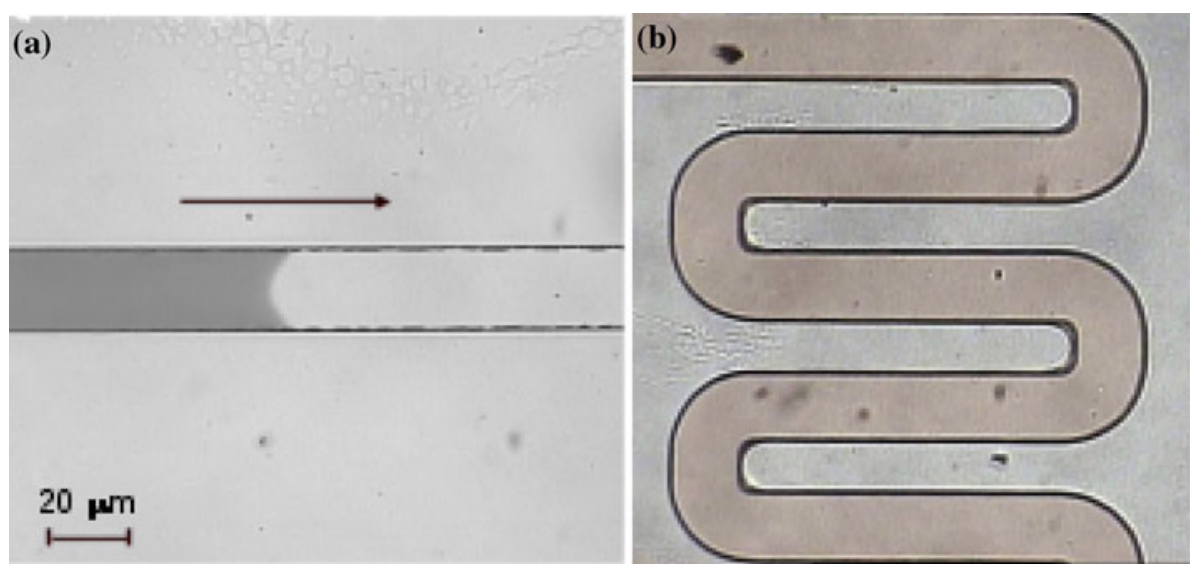

The microfluidic channel with micropillars shown in Fig. 9 is typical of the structures which have been used in this study to assess the volumetric flow rate of fluid flowing under capillary force in hybrid sol-gel microfluidic channels. This image shows a well-formed structure with sharp sidewalls, clean etching of the channel bottom surface and well-defined micropillars. Åmic AB (Uppsala, Sweden) has developed a new microfluidic test chip based on micropillar arrays similar to those shown in Fig. 9. The Åmic platform is intended for use in sandwich immunoassays and is fabricated by injection molding of the cycloolefincopolymer Zeonor $^{\mathrm{TM}}$. Recently, hybrid sol-gel materials have been incorporated into the fabrication of micro/nanopillar structures (Faustini et al. 2010). However, to the best of the knowledge, the study reported here is the first demonstration of micropillar arrays fabricated by the direct photopatterning of a photocurable hybrid sol-gel thin film. In light of advances made in the synthesis of non-shrinking photocurable hybrid sol-gel materials (Ovsianikov et al. 2008) and associated microfabrication techniques (Serbin

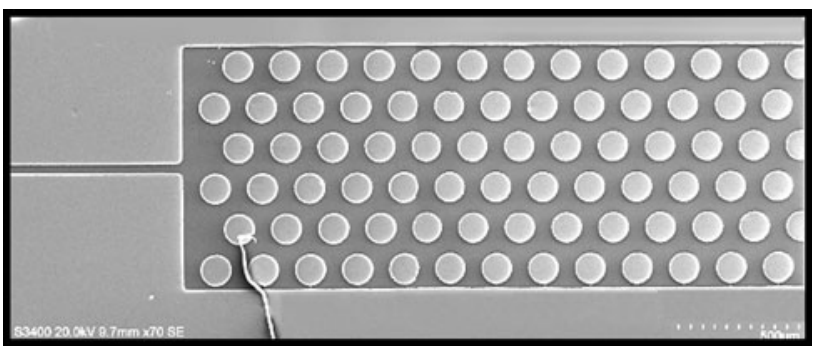

Fig. 9 SEM image of narrow microfluidic channel $(50 \mu \mathrm{m})$ coupled to a wide channel $(900 \mu \mathrm{m})$ filled with micropillars

et al. 2003; Ovsianikov et al. 2007), it is expected that photocurable hybrid sol-gel materials will receive renewed attention for the fabrication of a range of micropillar-based optical and/or microfluidic platforms including platforms for liquid chromatography (De Malsche et al. 2007).

The use of micropillars was critical for the formation of wide channels (where the aspect ratio was $\sim 100: 1$ ) as it prevented the PDMS cap layer from collapsing into the 
Fig. 10 Fluid flow in micropillar supported channel after: a $0 \mathrm{~s}$, b $0.12 \mathrm{~s}, \mathbf{c} 0.64 \mathrm{~s}$, and $\mathbf{d} 1.36 \mathrm{~s}$

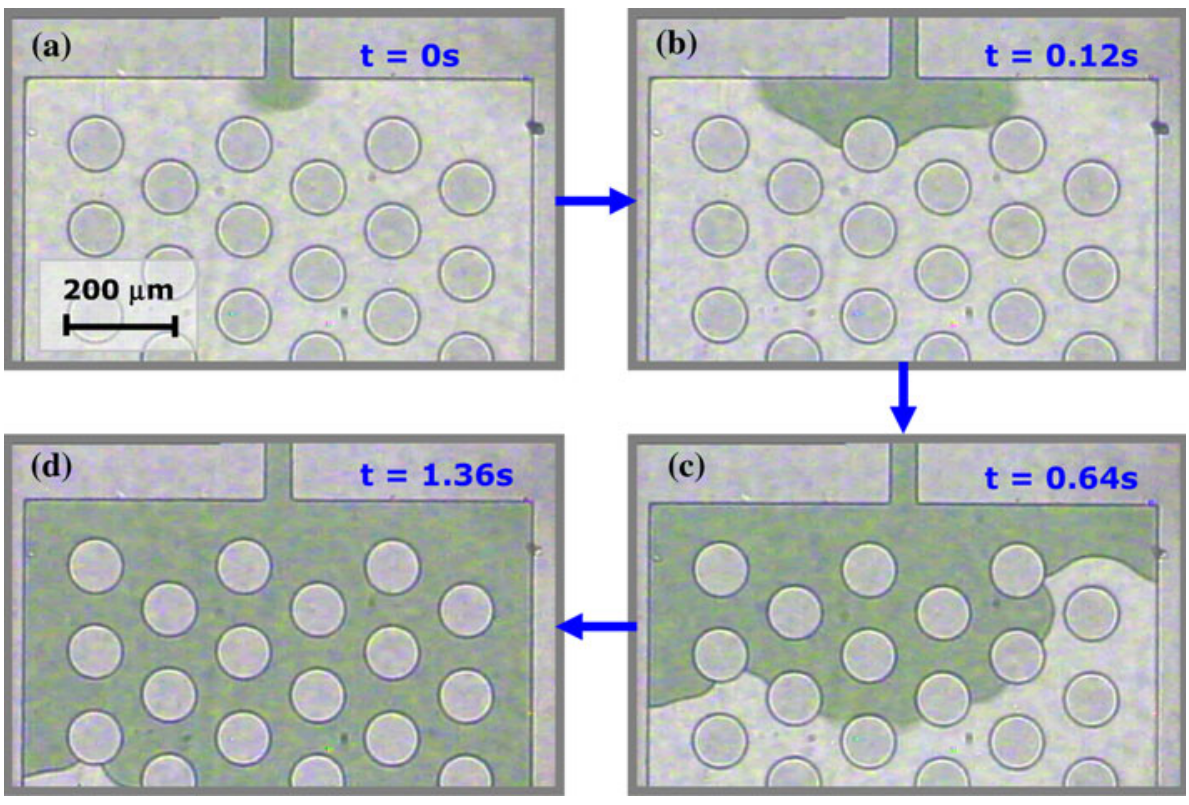

channel, thereby causing a blockage by bonding to the channel bottom surface. Images of fluid flow in this channel are displayed in Fig. 10 which shows the evolution over time of fluid flowing from the narrow input channel (at top of images in Fig. 10) into the wider micropillar array area. Examination of these images shows that when the plug of liquid transitions from the input channel to the micropillar array, the initially uniform and symmetrical meniscus profile degrades slightly as the fluid spreads to fill the micropillar array area. This is attributed to small fabrication inaccuracies, possibly originating from nonuniform oxygen plasma treatment of the channel surface. However, the meniscus profile did not degrade further as fluid flowed the full length of the $12 \mathrm{~mm}$ channel, with no more than $\sim 150 \mu \mathrm{m}$ separation noted between the leading and following edges of the meniscus. Progress of the leading edge of the meniscus was examined and quantified as shown in Fig. 11. It can be observed from these data that the rate of filling of the channel closely follows a linear trend line $\left(R^{2}=0.99\right)$ over time, with the slope of the linear fit indicating a channel filling speed of $0.0028 \mu \mathrm{l} / \mathrm{s}$ or $0.0168 \mu \mathrm{l} / \mathrm{min}$. This level of flow is in the typical region of reported values for capillary driven flow rates measured in microchannels (Bao and Harrison 2006; Lynn and Dandy 2009). The error bars indicate the influence that uncertainty in the meniscus position (estimated as $\pm 75 \mu \mathrm{m}$ ) has on calculation of the volume of channel filled with fluid.

The volumetric flow rate of fluid was also characterized in a number of other similar channels of differing micropillar radius and separation using the same methodology. The data presented in Fig. 12 shows that the volumetric flow rate of all channels tested varies between 0.12 and $0.18 \mu \mathrm{l} / \mathrm{min}$, which indicates that fluid flow in all channels

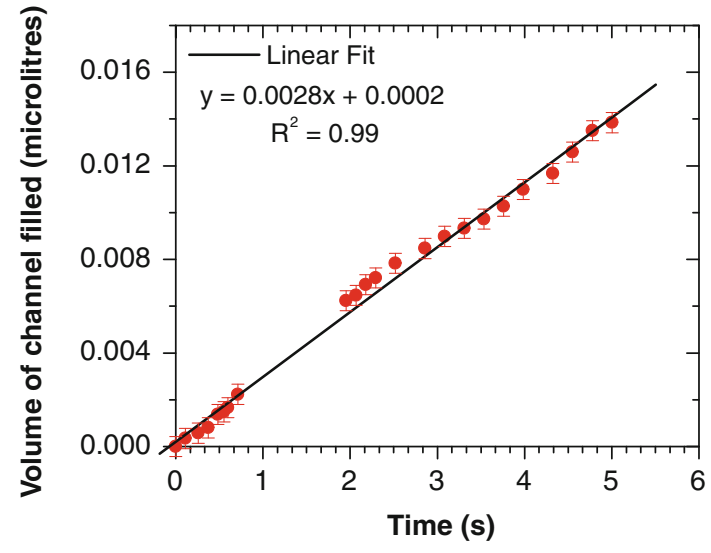

Fig. 11 Volume of channel filled with fluid as a function of time

was within typical boundaries for capillary-driven flow. For any given micropillar separation, a larger micropillar radius is seen to result in slightly larger volumetric flow rates. Owing to rapid hydrophobic recovery of surfaces directly after plasma treatment it is difficult to draw solid conclusions from the data gathered concerning the influence of channel dimension, micropillar radius and separation. Flow rates in microchannels were also tested one full day after plasma treatment and bonding with PDMS, and results reveal a significant slowing of fluid flow rate compared to testing directly after bonding, which is expected in the context of rapid hydrophobic recovery during the hours after oxygen plasma modification.

Bonding of all layers within a microfluidic device is a key factor when evaluating any new materials or fabrication processes. Examination of Fig. 10 shows that no leakage of the aqueous dye solution occurred into areas outside of the channel boundaries indicating that the cap 


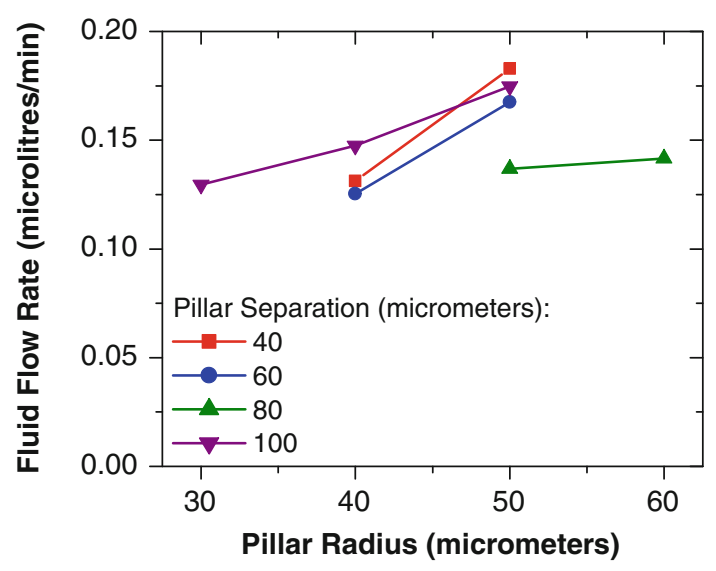

Fig. 12 Preliminary data showing the average flow-rate through a variety of channels containing micropillars of different radius (with different separations between micropillars)

and patterned sol-gel layer were sealed properly. This study verifies that after oxygen plasma treatment of the PDMS cap layer and photopatterned hybrid sol-gel microfluidic structures, irreversible bonds were formed between both layers which were sufficient to allow defect free bonding. In addition, it is possible to see that the PDMS cap did not collapse into the channel at any point due to the support provided by the micropillars.

In Fig. 13, images of the proof-of-concept structure are shown, which was fabricated according to the protocol outlined in Fig. 2. Fluid flowed rapidly through this channel with the concave meniscus profile characteristic of fluid flow in hydrophilic microchannels. This demonstrates that the presence of a channel waveguide in the path of a microchannel does not cause an obstruction as the fluid flowed freely without any observed resistance. In the context of immunoassay protocols, it is speculated here that a narrowing of the channel in the region of a sensor spot could have a positive influence on assays by lowering assay times. By narrowing the channel, a bottleneck in the channel is created thus forcing a greater interaction to occur between the target molecules (antigen) dispersed in the sample and the sensor spot (composed of capture antibodies). This should have the effect of increasing the chances of binding, which can decrease limits of detection.
In order to further progress this research, it is proposed to investigate the formation of thicker photopatternable films enabling a greater control over the aspect ratio of the microfluidic channels. Also, the composition of the hybrid sol-gel material is being investigated to find alternative precursors that result in the formation of intrinsically more hydrophilic materials. The study reported in this article is the first demonstration of the integration of microfluidic and photonic technologies fabricated from photocuarable hybrid sol-gel materials. Both surface and optical properties of the photocurable hybrid sol-gel materials have been shown to be highly tunable which is extremely promising for the fabrication of integrated microfluidics on photonic platforms.

\section{Conclusion}

If waveguide-based platforms are to gain widespread use for lab-on-a-chip (LoC) applications, microfluidic functionality needs to be integrated with the photonic element of the platforms in a low-cost process. To address these challenges, this article establishes a new fabrication route based on photocurable hybrid organic-inorganic materials. Significant results on the surface properties of such materials have been generated in this article, clearly identifying the routes by which a typical sol-gel material may be optimized to create hydrophilic surfaces capable of supporting capillary-driven fluid flow in microfluidic channels. Using these materials, a photolithographic fabrication process has been established which permits high accuracy patterning of the waveguide protective layer. Microchannels and micropillar arrays were fabricated and characterized and it was found that volumetric fluid flow rates varied between 0.012 and $0.018 \mu \mathrm{l} / \mathrm{min}$ which are characteristic of capillary-driven fluid flow. Furthermore, a proof of concept structure was fabricated in which a microchannel crossed a planer optical waveguide. Unimpeded fluid flow in the channel demonstrated that the presence of optical waveguides does not significantly disrupt capillary-driven fluid flow. These results represent the first comprehensive evaluation of hybrid sol-gel materials for use in optofluidic platforms.
Fig. 13 Image of capillary driven fluid flow in a hydrophilic microchannel intersecting an optical waveguide before (a) and after (b) flowing over the waveguide

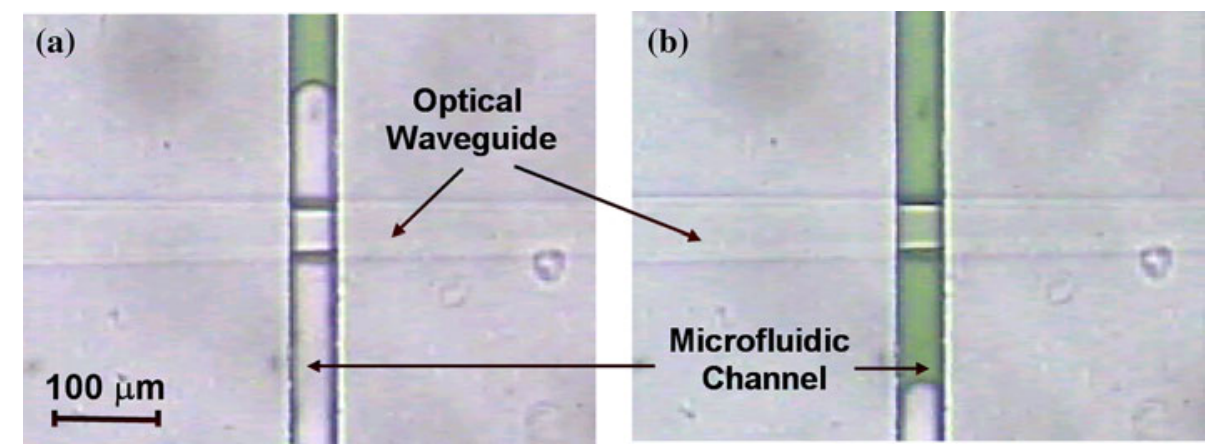


Acknowledgments The authors wish to thank CIBA Specialty Chemicals Corporation for having generously provided the Irgacures photoinitiators employed in this study, and gratefully acknowledge the financial support of Enterprise Ireland under the "Commercialisation Fund Technology Development" project Multibiosense (TD/ 08/309).

\section{References}

Avellaneda CO, Dahmouche K, Bulhoes LOS (2002) All sol-gel electrochromic smart windows: $\mathrm{CeO}_{2}-\mathrm{TiO}_{2} /$ Ormolyte. Mol Cryst Liquid Cryst 374:113-118

Avendano E, Berggren L, Niklasson GA, Granqvist CG, Azens A (2006) Electrochromic materials and devices: brief survey and new data on optical absorption in tungsten oxide and nickel oxide films. Thin Solid Films 496(1):30-36

Bao JB, Harrison DJ (2006) Measurement of flow in microfluidic networks with micrometer-sized flow restrictors. AICHE J 52(1):75-85

Basabe-Desmonts L, Benito-Lopez F, Gardeniers HJGE, Duwel R, van den Berg A, Reinhoudt DN, Crego-Calama M (2008) Fluorescent sensor array in a microfluidic chip. Anal Bioanal Chem 390(1):307-315

Becker H, Heim U (2000) Hot embossing as a method for the fabrication of polymer high aspect ratio structures. Sensors Actuators A Phys 83(1-3):130-135

Besanger TR, Brennan JD (2006) Entrapment of membrane proteins in sol-gel derived silica. J Sol-Gel Sci Technol 40(2-3):209-225

Bhattacharya S, Datta A, Berg JM, Gangopadhyay S (2005a) Studies on surface wettability of poly(dimethyl) siloxane (PDMS) and glass under oxygen-plasma treatment and correlation with bond strength. J Microelectromech Sys 14(3):590-597

Bhattacharya S, Datta A, Berg JM, Gangopadhyay S (2005b) Studies on surface wettability of poly(dimethyl) siloxane (PDMS) and glass under oxygen-plasma treatment and correlation with bond strength. J Microelectromech Syst 14(3):590-597

Birnie DP (2004) Surface skin development and rupture during solgel spin-coating. J Sol-Gel Sci Technol 31(1-3):225-228

Buso D, Della Giustina G, Brusatin G, Guglielmi M, Martucci A, Chiasera A, Ferrari M, Romanato F (2009) Patterning of sol-gel hybrid organic-inorganic film doped with luminescent semiconductor quantum dots. J Nanosci Nanotechnol 9(3):1858-1864

Copperwhite R, Oubaha M, Versace DL, Croutxé-Barghorn C, MacCraith BD (2008) The role of photoinitiator and chelating agent in the fabrication of optical waveguides from UVphotocurable organo-mineral sol-gel materials. J Non-Cryst Solids 354(30):3617-3622

Coudray P, Etienne P, Porque J, Moreau Y, Najafi SI (1998) Integrated optical devices achieved by sol-gel process, integrated optics devices II. The International Society for Optical Engineering, San Jose, p 252

De Malsche W, Clicq D, Verdoold V, Gzil P, Desmet G, Gardeniers $\mathrm{H}$ (2007) Integration of porous layers in ordered pillar arrays for liquid chromatography. Lab Chip 7(12):1705-1711

Edwards TL, Mohanty SK, Edwards RK, Thomas CL, Frazier AB (2002) Rapid micromold tooling for injection molding microfluidic components. Sensors Mater 14(3):167-178

Efimenko K, Wallace WE, Genzer J (2002) Surface modification of sylgard-184 poly(dimethyl siloxane) networks by ultraviolet and ultraviolet/ozone treatment. J Colloid Interf Sci 254(2):306-315

Everaert EP, Vandermei HC, Devries J, Busscher HJ (1995) Hydrophobic recovery of repeatedly plasma-treated siliconerubber.1. Storage in air. J Adhes Sci Technol 9(9):1263-1278
Everaert EP, VanderMei HC, Busscher HJ (1996) Hydrophobic recovery of repeatedly plasma-treated silicone rubber.2. A comparison of the hydrophobic recovery in air, water, or liquid nitrogen. J Adhes Sci Technol 10(4):351-359

Faustini M, Vayer M, Marmiroli B, Hillmyer M, Amenitsch H, Sinturel C, Grosso D (2010) Bottom-up approach toward titanosilicate mesoporous pillared planar nanochannels for nanofluidic applications. Chem Mater 22(20):5687-5694

Fois E, Gamba A, Tabacchi O (2008) Influence of silanols condensation on surface properties of micelle-templated silicas: a modelling study. Microporous Mesoporous Mater 116(1-3):718-722

Fujii T (2002) PDMS-based microfluidic devices for biomedical applications. Microelectron Eng 61-2:907-914

Gupta R, Chaudhury NK (2007) Entrapment of biomolecules in solgel matrix for applications in biosensors: problems and future prospects. Biosens bioelectron 22(11):2387-2399

Hamdy AS (2006) Advanced nano-particles anti-corrosion ceria based sol gel coatings for aluminum alloys. Mater Lett 60(21-22): 2633-2637

Hannes B, Vieillard J, Chakra EB, Mazurczyk R, Mansfield CD, Potempa J, Krawczyk S, Cabrera M (2008) The etching of glass patterned by microcontact printing with application to microfluidics and electrophoresis. Sensor Actuators B Chem 129(1): 255-262

Higgins C, Wencel D, Burke CS, MacCraith BD, McDonagh C (2008) Novel hybrid optical sensor materials for in-breath O-2 analysis. Analyst 133(2):241-247

Ho JA, Zeng S, Tseng W, Lin Y, Chen C (2008) Liposome-based immunostrip for the rapid detection of Salmonella. Anal Bioanal Chem 391(2):479-485

Hwang T, Lee H, Kim H, Kim G (2010) Two layered silica protective film made by a spray-and-dip coating method on 304 stainless steel. J Sol-Gel Sci Technol 55(2):207-212

Iliescu C (2006) Microfluidics in glass: technologies and applications. Informacije Midem-J Microelect Elect Comp Mater 36(4): 204-211

Jaynes WF, Boyd SA (1991) Hydrophobicity of siloxane surfaces in smectites as revealed by aromatic hydrocarbon adsorption from water. Clays Clay Mineral 39(4):428-436

Jiang HW, Kakkar AK (1999) An alternative route based on acid-base hydrolytic chemistry to NLO active organic-inorganic hybrid materials for second-order nonlinear optics. J Am Chem Soc 121(15):3657-3665

Karuppasamy A, Subrahmanyam A (2007) Studies on electrochromic smart windows based on titanium doped WO3 thin films. Thin Solid Films 516(2-4):175-178

Kribich KR, Barry H, Copperwhite R, Kolodziejczyk B, O’Dwyer K, Sabattie JM, MacCraith BD (2004) Thermo-optic switches using sol-gel processed hybrid materials. Proc SPIE 5451:518

Kribich K, Copperwhite R, Barry H, Kolodziejczyk B, Sabattie JM, O'Dwyer K, MaCCraith B (2005) Novel chemical sensor/ biosensor platform based on optical multimode interference (MMI) couplers. Sens Actuators B Chem 107(1):188-192

Lei BF, Li B, Zhang HR, Lu SZ, Zheng ZH, Li WL, Wang Y (2006) Mesostructured silica chemically doped with Ru-II as a superior optical oxygen sensor. Adv Func Mater 16(14):1883-1891

Liu RH, Stremler MA, Sharp KV, Olsen MG, Santiago JG, Adrian RJ, Aref H, Beebe DJ (2000) Passive mixing in a three-dimensional serpentine microchannel. J Microelectromech Sys 9(2):190-197

Lynn NS, Dandy DS (2009) Passive microfluidic pumping using coupled capillary/evaporation effects. Lab Chip 9(23):3422-3429

Mescher MJ, Swan EEL, Fiering J, Holmboe ME, Sewell WF, Kujawa SG, McKenna MJ, Borenstein JT (2009) Fabrication methods and performance of low-permeability microfluidic components for a miniaturized wearable drug delivery system. J Microelectromech Syst 18(3):501-510 
Najafi SI, Touam T, Sara R, Andrews MP, Fardad MA (1998) Sol-gel glass waveguide and grating on silicon. J Lightwave Technol 16(9): 1640-1646

Oubaha M, Smaihi M, Etienne P, Coudray P, Moreau Y (2003) Spectroscopic characterization of intrinsic losses in an organicinorganic hybrid waveguide synthesized by the sol-gel process. J Non-Cryst Solids 318(3):305-313

Oubaha M, Kribich R, Copperwhite R, Etienne P, OÕDwyer K, MacCraith B, Moreau Y (2005) New organic inorganic sol-gel material with high transparency at $1.55 \mathrm{~lm}$. Opt Commun 253: 346-351

Oubaha M, Copperwhite R, Murphy B, Kolodziejczyk B, Barry H, O'Dwyer K, MacCraith BD (2006) Development of photopatternable organo-mineral hybrid films from the sol-gel condensation of alkoxysilanes. Thin Solid Films 510(1-2): 334-338

Ovsianikov A, Chichkov B, Mente P, Monteiro-Riviere NA, Doraiswamy A, Narayan RJ (2007) Two photon polymerization of polymer-ceramic hybrid materials for transdermal drug delivery. Int J Appl Ceramic Technol 4(1):22-29

Ovsianikov A, Viertl J, Chichkov B, Oubaha M, MacCraith B, Sakellari L, Giakoumaki A, Gray D, Vamvakaki M, Farsari M, Fotakis C (2008) Ultra-low shrinkage hybrid photosensitive material for two-photon polymerization microfabrication. Acs Nano 2(11):2257-2262

Porque J, Coudray P, Charters R, Kribich K, Etienne P, Moreau Y (2000) WDM based on multimode interference-coupler built in an organic-inorganic material. Opt Commun 183(1-4):45-49

Serbin J, Egbert A, Ostendorf A, Chichkov BN, Houbertz R, Domann G, Schulz J, Cronauer C, Frohlich L, Popall M (2003) Femtosecond laser-induced two-photon polymerization of inorganic-organic hybrid materials for applications in photonics. Opt Lett 28(5):301-303

Varma PCR, Colreavy J, Cassidy J, Oubaha M, McDonagh C, Duffy B (2010) Corrosion protection of AA 2024-T3 aluminium alloys using 3,4-diaminobenzoic acid chelated zirconium-silane hybrid sol-gels. Thin Solid Films 518(20):5753-5761

Versace DL, Oubaha M, Copperwhite R, Croutxé-Barghorn C, MacCraith BD (2008) Waveguide fabrication in UV-photocurable sol-gel materials: influence of the photoinitiating system. Thin Solid Films 516(18):6448-6457

Zhang HX, Lu D, Fallahi M (2004) Active organic-inorganic sol gel with high thermal stability for nonlinear optical applications. Appl Phys Lett 84(7):1064-1066

Zhu Y, Petkovic-Duran K (2010) Capillary flow in microchannels. Microfluid Nanofluid 8(2):275-282 